\title{
Article \\ Development of a Pre-Evaluation and Health Monitoring System for FAST Cable-Net Structure
}

\author{
Yuzhou Shen ${ }^{1}\left(\mathbb{D}\right.$, Bin Luo $^{1, *}$, , Peng Jiang ${ }^{2}$, Mingmin Ding ${ }^{3}$, Qingwei Li $^{2}$ and Yang Wei ${ }^{3}(\mathbb{D}$ \\ 1 National Prestress Engineering Research Center, Key Laboratory of C \& PC Structures, Ministry of Education, \\ Southeast University, Nanjing 210096, China; yzshen@seu.edu.cn \\ 2 CAS Key Laboratory of FAST, National Astronomical Observatories, Chinese Academy of Sciences, \\ Beijing 100101, China; pjiang@nao.cas.cn (P.J.); qwli@nao.cas.cn (Q.L.) \\ 3 College of Civil Engineering, Nanjing Forestry University, Nanjing 210037, China; \\ dmm1989@njfu.edu.cn (M.D.); wy78@njfu.edu.cn (Y.W.) \\ * Correspondence: seurobin@seu.edu.cn
}

check for

updates

Citation: Shen, Y.; Luo, B.; Jiang, P.;

Ding, M.; Li, Q.; Wei, Y. Development of a Pre-Evaluation and Health Monitoring System for FAST Cable-Net Structure. Appl. Sci. 2022 12, 332. https://doi.org/10.3390/ app12010332

Academic Editors: Evangelos I. Katsanos, Sandro D. R. Amador, Isaac Farreras Alcover

Henrik Gjelstrup and Rune Brincker

Received: 7 December 2021

Accepted: 29 December 2021

Published: 30 December 2021

Publisher's Note: MDPI stays neutral with regard to jurisdictional claims in published maps and institutional affiliations.

Copyright: (C) 2021 by the authors. Licensee MDPI, Basel, Switzerland. This article is an open access article distributed under the terms and conditions of the Creative Commons Attribution (CC BY) license (https:// creativecommons.org/licenses/by/ $4.0 /)$.

\begin{abstract}
The Five-hundred-meter Aperture Spherical radio Telescope (FAST) is one of China's major pieces of national infrastructure. A variable cable-net structure is used as the main supporting structure of the active reflector. The displacement of the cable net works through actuators. The realization of linkage control is a multi-degree-of-freedom and complex coupling control system. Due to factors such as the temperature difference between day and night, as well as actuator failure, the reflector control accuracy and even structural safety are affected during the position-control process of the cable net, so realizing evaluation of control accuracy and fault warning of the reflector is a significant problem. This paper proposes a pre-evaluation and health monitoring system based on advanced mechanical simulation technology. Through this system, on-site staff can expeditiously analyze the model to determine whether the cable net is currently in a safe state, predict the fatigue degree of the components, and maintain the structure when appropriate. The pre-evaluation and health monitoring system adequately ensure the stable functioning of the FAST cable net, improve the efficiency of on-site maintenance work, and markedly reduce the safety risk of the structure.
\end{abstract}

Keywords: radio telescope; cable-net structure; health monitoring; mechanical simulation technology; analytical methods

\section{Introduction}

The Five-hundred-meter Aperture Spherical radio Telescope (FAST) belongs to China's national "Eleventh Five-Year Plan" major infrastructure construction projects and is now the largest single-aperture radio telescope in the world [1]. The construction of FAST was completed in 2016 and has been officially put into use since 2020. Its reflector is approximately 30 football fields in size, and its sensitivity is more than 2.5 times that of the world's second largest telescope. Its advanced design ideas, coupled with the advantage of its unique site, ensures that FAST will be in the world's leading position for the next 30 years [2].

Active displacement is the most prominent feature of the FAST reflector. FAST uses the overall cable-net structure as the supporting structure of the reflector (see Figure 1). The overall cable-net structure comprises a main cable net, pull-down cables, and a peripheralring support structure [3]. The 6670 main cables are woven into a spherical surface in a grid manner, forming 2225 nodes. Each node connects a pull-down cable. The lower ends of these pull-down cables are combined with ground actuators to control the displacement of the entire cable net. The peripheral ring supporting the structure includes a ring beam and steel pillars, and the inner side of the ring beam is connected to the main cables by lug-plate joints. The inner diameter of the ring beam is $500.8 \mathrm{~m}$, while the total weight is 5350 tons, and it is composed of a ring truss with dimensions of $11 \mathrm{~m} \times 5.5 \mathrm{~m}$ [4]. The ring 
beam is located on sliding bearings and sustained by 50 steel columns of differing heights. Displacement of the cable net is realized by the linkage control of 2225 actuators, which is essentially a multi-degree-of-freedom, complex coupled control system [5].

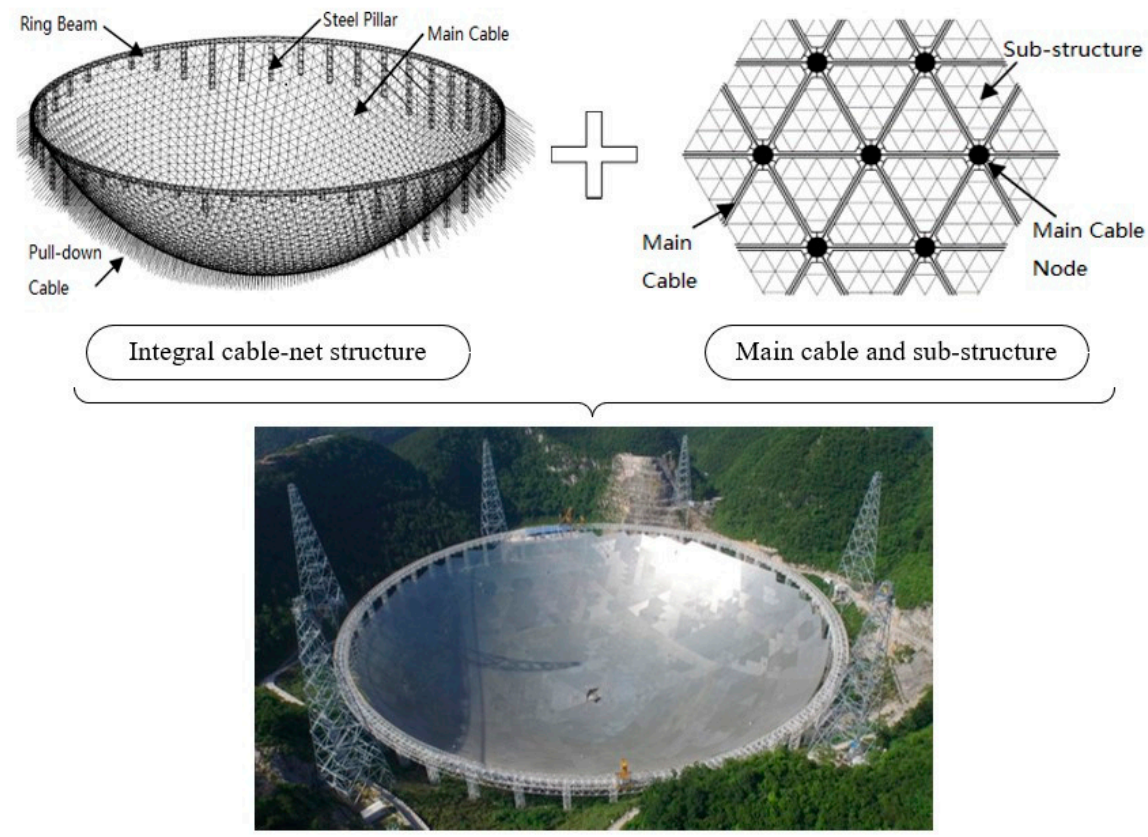

Figure 1. Sketch of the FAST cable-net structure.

Different from cable-net structures in the general architectural field, FAST has a particular use function as an astronomical radio telescope. Playing the most crucial role in FAST displacement, the cable net has an ultralong span and flexible shape. Its active reflector state includes two main parts: (1) The basic spherical state. The supporting structure of the FAST active reflector is subject to its own weight (including the weight of the back frame structure and the cable-net structure) and pre-stressing force. The main cable-net nodes are in the equilibrium state on the designated reference sphere, which is the basic spherical state. (2) Active displacement working state. In order to meet the needs of astronomers for observing celestial bodies in different directions and distances, FAST needs to form paraboloids of various calibres at particular angles, and this function is achieved by the actuators dragging the pull-down cables (See Figure 2) [6,7].

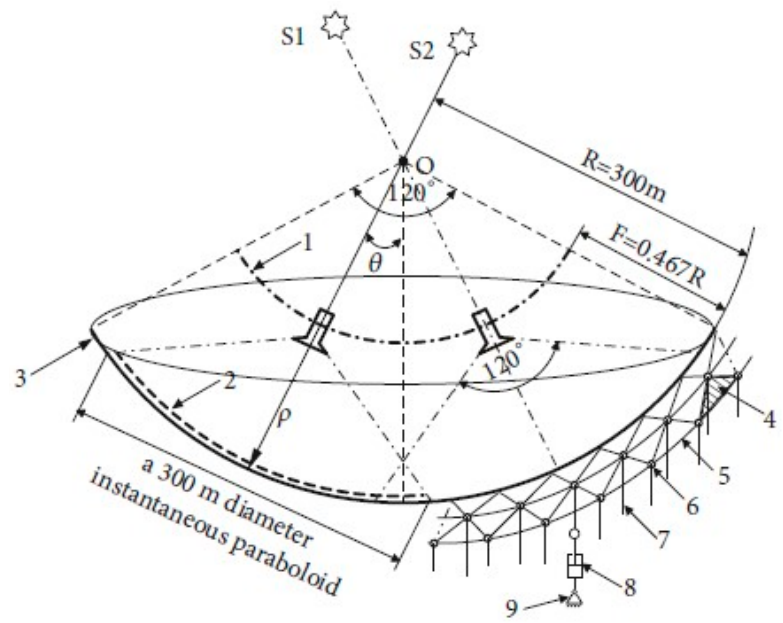

Figure 2. Schematic diagram of the FAST reflector. 1: focusing surface; 2: paraboloid form; 3: basic spherical surface; 4: reflector panel; 5: main cable; 6: cable-net node; 7: pull-down cable; 8: hydraulic actuator; 9: anchor block. 
FAST faced various disadvantages and risks during its design period. Although detailed analyses were carried out at that time, they were based on the initial design model. FAST has a design life of at least 30 years, which means that the design model will necessarily change during actual operation [8]. For example, (1) FAST uses giant, natural karst depressions on the earth as the telescope site, which are easy to collapse and erode, resulting in insufficient foundations. (2) FAST is located in a humid and rainy area. Chronic rainfall and stagnant water can easily cause uneven structural forces. (3) Certain actuators are prone to failure once under high-intensity observation, which could cause the actual force state to be inconsistent with the theoretical state. Even in the most unfavourable case, the cables connected to these ineffective actuators will bear loads that exceed their ultimate bearing capacity and eventually lead to the striking of nearby main cables [9].

The FAST cable net needs to be deformed frequently when undertaking long-term and arduous observation tasks, which makes the cable net bear cyclically changing loads for long periods, which will eventually lead to the fatigue and failure of cables. The FAST reflector realizes displacement drive by controlling the actuators to stretch or relax the pull-down cables. The displacement work is essentially a long-term fatigue load. Therefore, the fatigue life of the cable net under long-term displacement is a critical factor in determining whether FAST can be successfully constructed. According to calculations, the maximum fatigue stress amplitude of the FAST cables is between 500 and $525 \mathrm{MPa}$, while standard cables generally do not exceed $300 \mathrm{MPa}$. FAST researchers invented a highstrength OVM.ST-type high-stress-amplitude cable system that meets the requirements of more than 30 years of durability, after quite a few years of indignation $[10,11]$.

Even so, the frequent variation of angles and calibres in daily tasks could still lead to the actual stress amplitude deviating from the designed stress amplitude, which is more likely to cause cables to be inconsistent with the designed fatigue condition. Moreover, once cables fail due to fatigue, they may cause damage to the reflector and actuators in the adjacent area, which could ultimately cause casualties. Furthermore, it is difficult for the cables to accurately reach the predetermined positions under the influence of fatigue failure; thus, the corresponding celestial-observation efficiency could be reduced.

In fact, the FAST cable net has nearly 10,000 cables with different areas and lengths, and these cables are all customized, so it is difficult to replace damaged cables at any time. Due to the great difficulty of producing these cables, once the cables are damaged, it may take quite a long time for them to be replaced. Consequently, it is imperative to realize in advance which cables will have fatigue failure and when. Over 500 sensors are installed on FAST at present, which still seems a little scarce in the face of around 10,000 cables supporting FAST. It is hard to effectively evaluate the safety of cable-net structures only by arranging these sensors [12].

Because there are many problems, such as the temperature difference between day and night and potential actuator failure during the operation of FAST, these factors will be coupled with one another in the process of cable-net displacement. At the same time, they present considerable randomness, which could significantly affect control accuracy and even the safety of the reflector. Therefore, realizing assessment of control accuracy and fault warning of the reflector is a significant problem faced by the telescope. Accordingly, the development of an efficient and stable FAST cable-net operation and maintenance technology is an urgent problem to be solved.

Zhu [13] proposed a method of using hydraulic-actuator data to determine whether there are structural safety issues. Still, it is difficult to determine the magnitude of the impact on structural accuracy. Zhu [14] put forward an approach of installing a large number of fibre Bragg grating sensors on the FAST cable net. The surface strain of the cable head is tested to indirectly obtain the cable-force value and adjust the model periodically. However, this method is inefficient and cannot meet the immediate requirements of reflector warnings. Fan [15] established an early-warning platform, which could provide timely feedback on the failure of the FAST active reflector. Still, it was unable to determine the gap 
between the actual stress change of the cable net and the theoretical stress spectrum, which would lead to fatigue risk for the cables in the cable net.

The safety early-warning system is an integral part of FAST health monitoring. It is an essential guarantee for the safe operation of the structure. The work efficiency of the traditional manual inspection method can no longer meet the requirements of the structural-safety early warning. Due to the enormous international influence of FAST, in order to avoid a tragedy such as that of the Arecibo telescope, its monitoring and earlywarning system must be able to detect danger accurately and effectively in the first place and promptly remind on-site staff to take necessary measures to avoid accidents.

This paper develops an early-warning system for FAST in terms of two parts, including pre-evaluation and health monitoring (PHM) systems. The model establishment parameters of the FAST reflector are studied, combined with mechanical simulation technology (MST), and an accurate model of the FAST reflector is established. The rainflow counting method is utilized to obtain the fatigue-stress amplitude curve of cables. The information is displayed in the system through MATLAB so that the pre-evaluation can be controlled visually. Development of the system has now been completed. The system can evaluate the safety condition of the FAST cable net at any time, promptly warn of possible accidents on the cable net, and predict the remaining working life of every cable, which effectively guarantees the stable operation of FAST. With these data, on-site staff can replace damaged components at the first opportunity. A field test also proved that the system effectively improved maintenance efficiency and reduced maintenance costs.

\section{Basic Concept of Mechanical Simulation Technology}

Mechanical simulation technology combines the real physical world with computers. By analysing the data periodically fed back by the equipment and the real-time data obtained during constant operation, MST can grasp the status of objects in the physical world in an accurate and timely manner. The development of computer technology in recent years has made it possible to establish a simulation model and environment for multi-dimensional information interaction. Unlike traditional simulation technology, MST can provide dynamic images based on real-time changing data and draw entities visually in the computer [16].

MST is emerging in the high-end industrial field. Many companies are keeping pace. For instance, an airline used MST for the health maintenance and sustainment of aerospace vehicles. They established a model of actual aeroplanes in computer and realized the actual state of the aeroplane through synchronization with sensors. By this means, whether the aeroplane needed maintenance or could withstand the next mission load could be analysed and evaluated in a timely manner according to the existing structure and past load after each flight. MST can integrate diachronic maintenance data and related derived data from the health monitoring system. By integrating the above data, MST can continuously monitor and forecast the health condition, the remaining working life, and the probability that the system will continue to work normally. It also indicates the response to critical security incidents of the system.

MST is used to collect data such as cable force and actuator stroke in the FAST cable net in our system, which not only meets the requirements of low data delay but also acquires enough data for real-time monitoring. Through the calculation, the safe operation status of FAST can be evaluated over time, warn of possible accidents on the structure in advance, and guarantee the stable operation of FAST.

\section{Establishment of the FAST MST Model}

\subsection{Method of Obtaining Data of the FAST Cable Net}

When establishing the pre-evaluation and health monitoring system, the first and most critical work is to establish an MST model consistent with the actual FAST cable-net structure. There are various sensors and other measuring instruments installed on all parts of the FAST cable net. For example, magnetostrictive sensors were set up on every actuator 
to obtain the strokes of the equipment; magnetic flux sensors for measuring cable force were installed on 150 edge main cables; the coordinates of lug-plate pinholes can be measured and recorded automatically by assigning reflected targets of the laser measurement to them; and so on [17]. The measuring equipment makes it possible to establish the MST model of the FAST cable net quickly and accurately.

ANSYS Parametric Design Language (APDL) is an advanced application of ANSYS that is widely used in engineering projects and has a powerful programming capability. It is beneficial to use APDL in establishing the MST model. The complete ANSYS model of the cable net, including cables, beams, and columns, is shown in Figure 3.

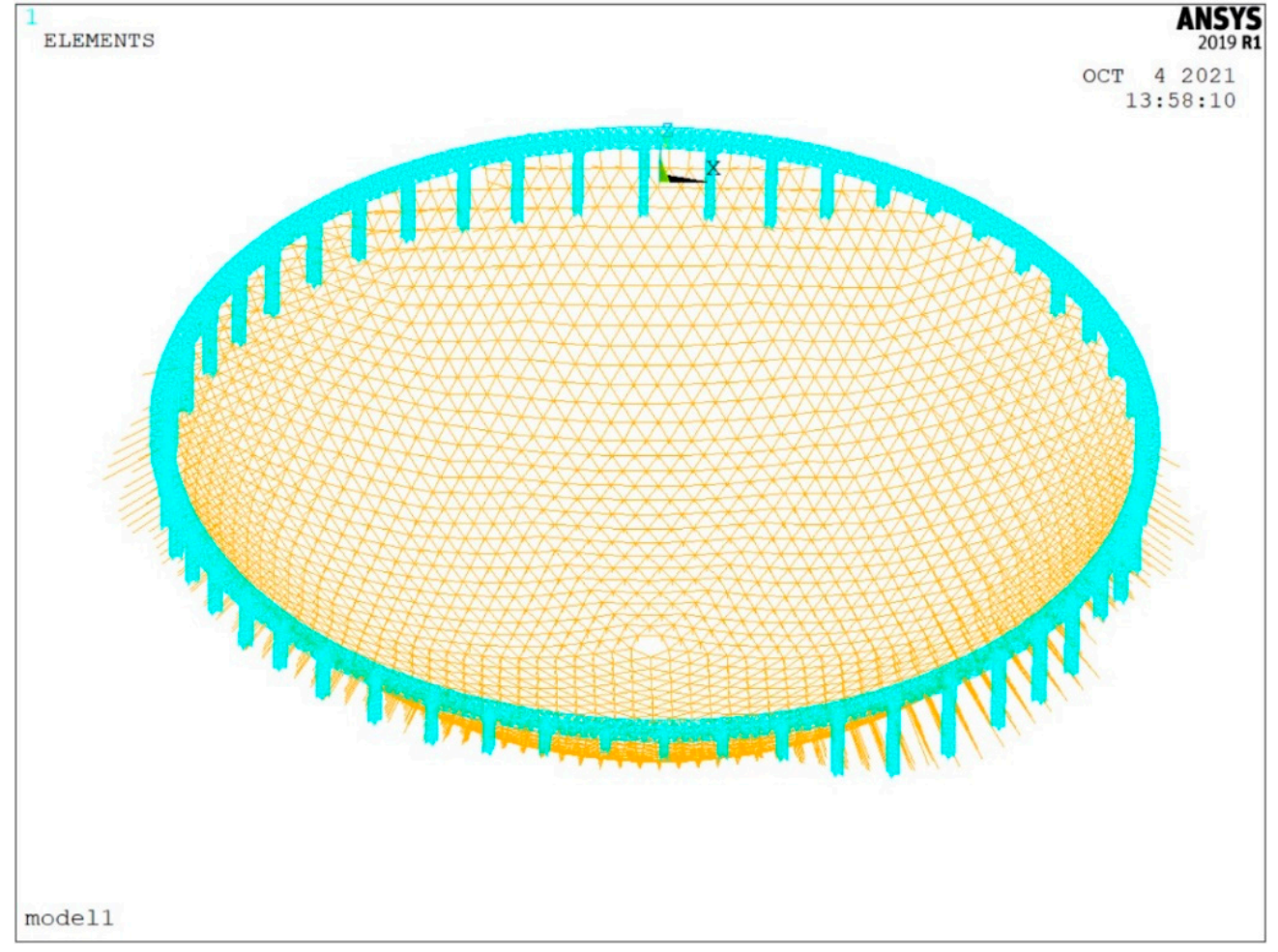

Figure 3. ANSYS model of FAST cable net.

The BEAM 44 element is a beam element that can withstand tension, compression, torsion, and bending, so we use it to simulate ring beams and pillar components. The LINK 180 element is a tension and compression element in the direction of the rod axis, which cannot bear the bending moment. It is used to simulate all the cables in the model. Because the cables are not compressed, they are set to be tension-only. The main subject of the MST analysis is the main cables and pull-down cables. While the requirements for the speed of analysis are significant, each cable is meshed into one element. The weight of the reflector panel is transformed to vertical loads in intersections of pull-down cables and main cables.

As mentioned above, MST data collection is based on the dynamic changes of previous and present data. In other words, when structural forces change, they need to be collected and transmitted to the model. According to the differing speeds of data change, data can be divided into two categories: regularly measured data and real-time collected data. The data measured regularly will not change in a short time period, so these data are usually updated to the MST model uniformly after the scheduled regular measurement. In contrast, the data collected in real time may change at any time, which has a more significant impact on the overall stress level of the FAST cable net and belongs to the type of data that is of crucial concern. Therefore, updating the real-time collected data in time is essential.

FAST adopts active reflector technology to observe celestial bodies by controlling the movement of the cable net to form a paraboloid, while the cable net shifts by means of changing the stroke of actuators. Therefore, ineffective actuators lead to loss of the 
working ability of the pull-down cables connected to them and cause the stress level of the corresponding main cables to be abnormal. Moreover, since FAST is located in a mountainous area, the surrounding environment has a large temperature difference with the massive volume of FAST, so the effect of temperature difference on the stress in the whole structure cannot be neglected. Hence, the number of ineffective actuators, the current strokes, and the ambient temperature in the mountains are the most important data need to be obtained during the daily work of FAST. Such data must be updated into the MST model without delay.

Real-time collected data are obtained through communication between MATLAB's OLE for process control module and a programmable logic controller. The data transmission time per cycle is generally $0.3 \sim 0.4 \mathrm{~s}$. Once the new data are transferred to the computer, they will be stored in the designated folder in the form of Excel, and the data from the previous round will be replaced. Therefore, ANSYS updates the MST model by invoking the Excel table file.

\subsection{Types and Parameters of FAST Cables and Connecting Nodes}

FAST uses the OVM.ST high-stress amplitude cable (see Figure 4), the main technical performance parameters of which are as follows:

(1) The pull-down cable uses specification No.1 in Table 1 (OVM.ST15-1), while the main cable uses specification Nos. 2-21 in Table 1.

(2) The elastic modulus of the cable: $\mathrm{E}=(190 \pm 10) \mathrm{GPa}$;

(3) The linear temperature expansion coefficient: $\alpha=1.2 \times 10^{-5}$;

(4) Other parameters of the cables are shown in Table 1.

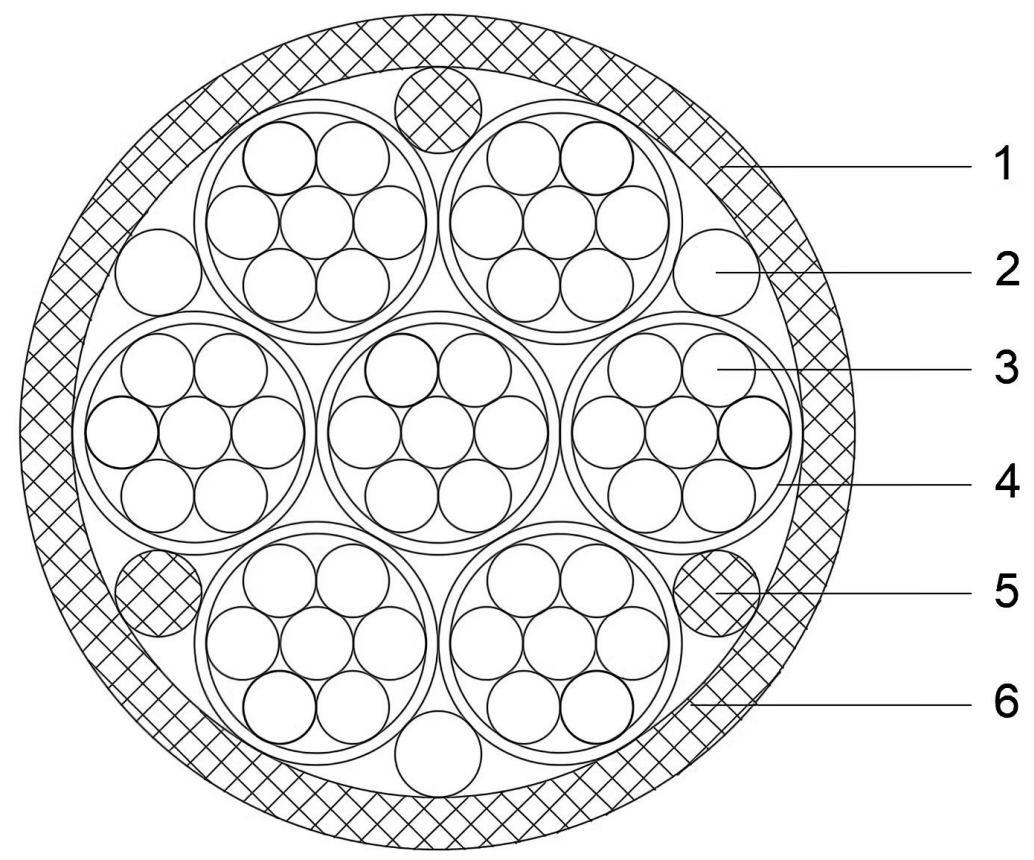

Figure 4. Sectional view of finished FAST cables. 1: Outer HDPE; 2: galvanized steel wire; 3 : epoxy-coated steel strand; 4: inner HDPE; 5: stuffing rope; 6: high-strength polyester tape. 
Table 1. Parameters of OVM.ST cable and connection nodes.

\begin{tabular}{|c|c|c|c|c|c|c|c|c|c|}
\hline \multirow{3}{*}{$\begin{array}{c}\text { Serial } \\
\text { Number }\end{array}$} & \multirow{3}{*}{ Specification } & \multicolumn{4}{|c|}{ Cable Body } & \multicolumn{2}{|c|}{$\begin{array}{c}\text { Double Cable } \\
\text { Heads }\end{array}$} & \multicolumn{2}{|c|}{ Connection Node } \\
\hline & & $\begin{array}{l}\text { Cross- } \\
\text { Sectional } \\
\text { Area }\end{array}$ & $\begin{array}{l}\text { Linear } \\
\text { Weight }\end{array}$ & $\begin{array}{c}\text { Outer } \\
\text { Diameter }\end{array}$ & $\begin{array}{l}\text { Limit Cable } \\
\text { Force }\end{array}$ & Weight & Length & Weight & $\begin{array}{c}\text { Outsourcing } \\
\text { Diameter of } \\
\text { Pinhole Centre }\end{array}$ \\
\hline & & $\mathrm{cm}^{2}$ & $\mathrm{~kg} / \mathrm{m}$ & $\mathrm{mm}$ & $\mathbf{k N}$ & kg & $\mathrm{mm}$ & kg & $\mathrm{mm}$ \\
\hline 1 & OVM.ST15-1 & 1.4 & 1.37 & 23 & 260 & 10.5 & 390 & - & - \\
\hline 2 & OVM.ST15-2 & 2.8 & 3.29 & 44 & 520 & 40 & 640 & 41 & 270 \\
\hline 3 & OVM.ST15-2J3 & 3.4 & 3.65 & 44 & 629.5 & 40 & 640 & 41 & 270 \\
\hline 4 & OVM.ST15-3 & 4.2 & 4.52 & 47 & 782 & 52 & 700 & 55 & 290 \\
\hline 5 & OVM.ST15-3J3 & 4.8 & 4.87 & 47 & 891.5 & 52 & 700 & 55 & 290 \\
\hline 6 & OVM.ST15-4 & 5.6 & 5.71 & 51 & 1040 & 70 & 800 & 90 & 360 \\
\hline 7 & OVM.ST15-4J3 & 6.2 & 6.07 & 51 & 1149.5 & 70 & 800 & 90 & 360 \\
\hline 8 & OVM.ST15-5 & 7 & 7.29 & 62 & 1300 & 87 & 830 & 115 & 390 \\
\hline 9 & OVM.ST15-5J3 & 7.6 & 7.75 & 62 & 1409.5 & 87 & 830 & 115 & 390 \\
\hline 10 & OVM.ST15-6 & 8.4 & 8.29 & 62 & 1560 & 92 & 880 & 132 & 400 \\
\hline 11 & OVM.ST15-6J3 & 9 & 8.75 & 62 & 1669.5 & 92 & 880 & 132 & 400 \\
\hline 12 & OVM.ST15-7 & 9.8 & 9.29 & 62 & 1820 & 108 & 880 & 172 & 450 \\
\hline 13 & OVM.ST15-7J3 & 10.4 & 9.75 & 62 & 1929.5 & 108 & 880 & 172 & 450 \\
\hline 14 & OVM.ST15-8 & 11.2 & 11.22 & 74 & 2080 & 151 & 1020 & 194 & 450 \\
\hline 15 & OVM.ST15-8J3 & 11.8 & 11.68 & 74 & 2189.5 & 151 & 1020 & 194 & 450 \\
\hline 16 & OVM.ST15-9 & 12.6 & 12.52 & 80 & 2340 & 185 & 1030 & 253 & 520 \\
\hline 17 & OVM.ST15-9J3 & 13.2 & 12.99 & 80 & 2449.5 & 185 & 1030 & 253 & 520 \\
\hline 18 & OVM.ST15-10 & 14 & 13.52 & 80 & 2600 & 199 & 1120 & 268 & 520 \\
\hline 19 & OVM.ST15-10J3 & 14.6 & 13.99 & 80 & 2709.5 & 199 & 1120 & 268 & 520 \\
\hline 20 & OVM.ST15-11 & 15.4 & 14.74 & 81 & 2860 & 212 & 1150 & 307 & 520 \\
\hline 21 & OVM.ST15-11J3 & 16 & 15.2 & 81 & 2969.5 & 212 & 1150 & 307 & 520 \\
\hline
\end{tabular}

\subsection{Update Process of MST Model}

The update procedure of MST model data will be described below. The stroke change of the actuators first needs to be updated into the model. The ambient temperature is used as the load condition for model calculation. The deformation of pull-down cables is equivalent to the strokes of connected actuators, which is converted into the temperature change of the pull-down cables and superimposed with the ambient temperature through Equation (1):

$$
\Delta T_{i}=\frac{\Delta L_{i}}{\alpha \times L_{i}}+T_{\mathrm{e}}
$$

Here, $\Delta T_{i}$ represents the final temperature change of the $i$ th pull-down cable. $\Delta L_{i}$ is the deformation of the $i$ th pull-down cable. $\alpha$ indicates the linear temperature expansion coefficient of the cable, which is $1.2 \times 10^{-5}$. $L_{i}$ is the length of the $i$ th pull-down cable in the model. $T_{e}$ stands for ambient temperature.

Ineffective actuators, based on different kinds of failure-response modes, can be divided into two situations: pulled out or stuck. The connected pull-down cable in the MST model would be disabled if the actuator was pulled out; when actuator is found stuck, the last stroke collected from the cycle is entered into the model.

Since FAST was completed and put into use, we have continued to measure the regularly measured data every six months, which include: (1) the coordinates of all lugplate pinholes, which are used to simulate the relative displacement between the edge main cable and the joints of the beam; (2) the coordinates of cross nodes between the main cables and pull-down cables under the basic spherical surface in a curvature radius of $300 \mathrm{~m}$ and the strokes of the actuators. These coordinates are used to check whether the cable net has 
an overall displacement. (3) The strokes and coordinates of all actuators can be checked to determine whether the positions of pull-down cables move or their lengths change.

The similarity between the regularly measured data and the real-time collected data is that they are all stored in Excel files, while the difference is that the regularly measured data are used to modify the geometry of the ANSYS model by invoking the APDL program. On the contrary, the data collected in real time are applied as loads in the model. After calculating and ensuring that the structure is in a safe state, the real-time data are overwritten by subsequent data. In that case, the model is not changed.

FAST is a statically multiple-indeterminate structure. The length and elastic modulus of the cables, the coordinates of the boundary nodes and actuators, and the tension of the active cable all change the internal force of the structure and affect the results of the simulation. Therefore, it is required to continuously iterate the parameters in the model by comparing the measured data with the results of theoretical analysis to make the model as close to the actual situation as possible.

Because the FAST basic sphere is the reference form of the deformation, the MST model is constantly updated in the state of the basic sphere. The update method of the MST model is shown below.

During the non-observation period of FAST, the basic sphere can be calibrated at the best working time of the total station on site through the automatic calibration system, which is usually 4 a.m. except in cases of inclement weather. The error between the calibrated basic sphere and the standard sphere shall be no more than $2 \mathrm{~mm}$. The number of ineffective actuators, all actuator strokes, and the ambient temperature can be acquired through on-site sensors. The coordinates of cross nodes, actuators and lug-plate pinholes, as well as the force of edge main cables, are also measured simultaneously in the case of an integral slide.

With the coordinates of actuators and lug-plate pinholes, the geometric shape of the MST model can be modified, and afterwards, finite element analysis can be performed on the model. For the cross nodes of main cables and pull-down cables, the deviation between the measured and analysed coordinates includes two parts: radial and tangential deviation.

For the pull-down cable, due to the existence of the cable head, assuming that the cable head is a rigid body, the converted elastic modulus of the pull-down cable can be calculated according to Equation (2).

$$
E_{i 0}=\frac{L_{i}}{L_{i}-L_{c h}} \times E_{\mathcal{c}}
$$

Here, $E_{i 0}$ stands for the converted elastic modulus of pull-down cables; $L_{i}$ is the length of the $i$ th pull-down cable in the model; $L_{c h}$ indicates the length of the cable head of the pull-down cable; and $E_{c}$ is the elastic modulus of the pull-down cable.

Since the cross nodes of the FAST cable net have little influence on one another, the radial deviation is mainly affected by the stiffness change of the pull-down cable. The FAST pull-down cable is long, which leads to a nonlinear increase in the sag of the pull-down cable, and the catenary effect cannot be ignored. As a consequence, it is necessary to calculate the elastic-modulus correction value for pull-down cables according to Equation (3):

$$
E_{i}=\frac{L_{i} \times F_{i}}{\Delta L_{i} \times A_{i}}+E_{i 0}
$$

where $E_{i}$ is the elastic modulus of the $i$ th pull-down cable after correction; $F_{i}$ represents the theoretical cable force of the $i$ th pull-down cable; $\Delta L_{i}$ indicates the radial deviation of the connected cross node; and $A_{i}$ stands for the cross-sectional area of the cable.

The main reason for the tangential deviation of the nodes is the strain of the main cable. From the coordinate changes of main cable nodes before and after the calculation, 
the length change of the main cable can be obtained. The strain of the main cable can be modified and updated in the MST model accordingly with Equation (4):

$$
\Delta \varepsilon_{j}=\frac{L_{j 1}-L_{j 2}}{L_{j 2}}
$$

Here, $\Delta \varepsilon_{j}$ is the strain-correction value of the $j$ th main cable; $L_{j 1}$ is the measurement length of the $j$ th main cable; and $L_{j 2}$ indicates the theoretical length, which can be calculated by the following equation:

$$
L_{j 2}=L_{c h}+L_{c b}+\left(B_{1}+B_{2}\right) / 2
$$

Here, $L_{c h}$ represents the length of the cable head of the main cable; $L_{c b}$ is the length of the cable body of the main cable; $B_{1}$ and $B_{2}$ are the outsourcing diameter of the pinhole centre of connectors at both ends, respectively, of the main cable from Table 1.

Through the above iterative method, the model can be continuously updated to narrow the gap between theoretical and measured values. For FAST, the error of the cross node is not allowed to be greater than $5 \mathrm{~mm}$. When all cross nodes meet this requirement, the model after the last iteration is regarded as usable. We habitually use the APDL language to compile the finite-element program so that the MST model can be automatically updated. A flow chart of the updating process is shown in Figure 5.

Real-time feedback data

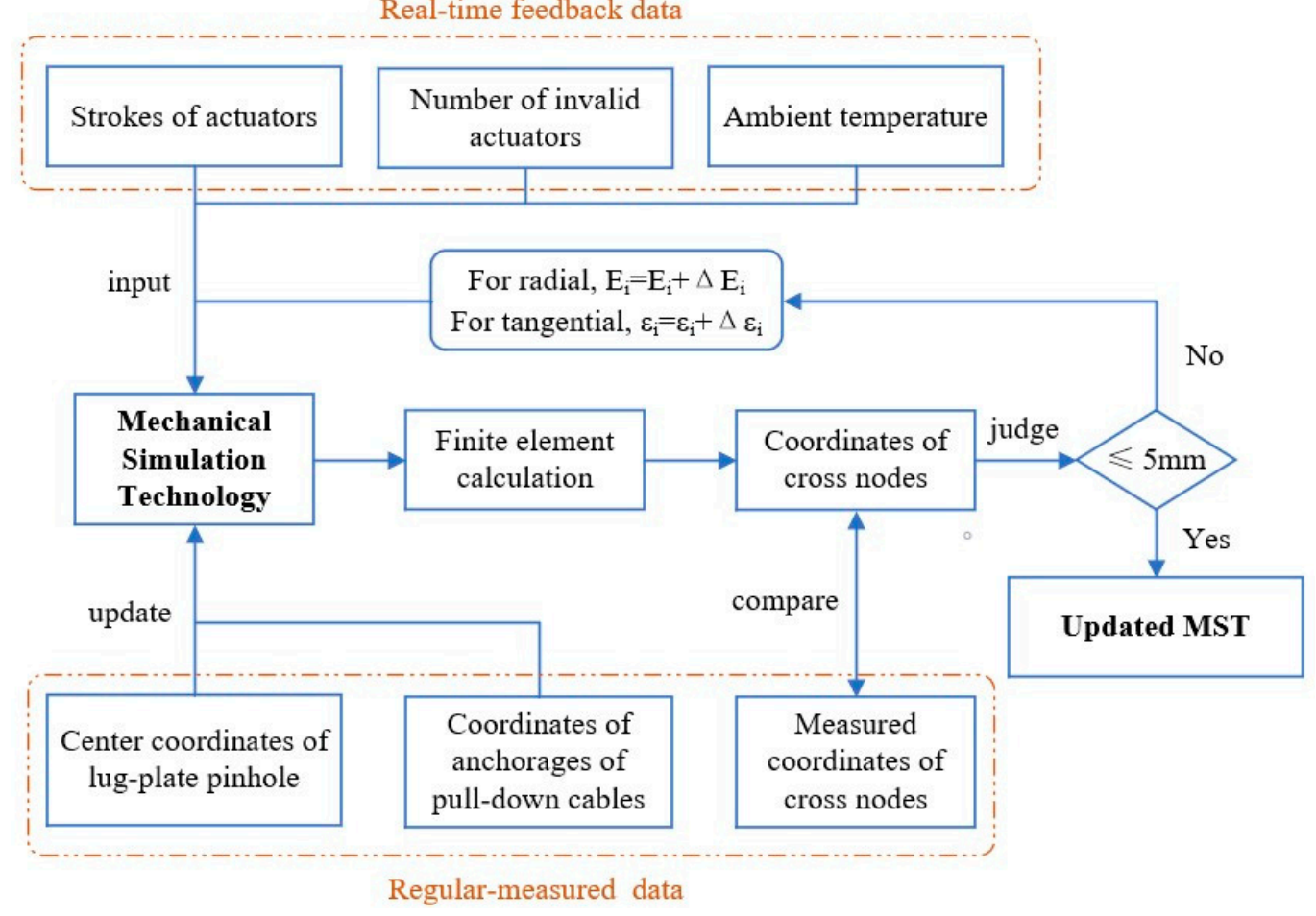

Figure 5. Flow chart of the updating process.

\subsection{Verification of the Accuracy of the MST Model}

FAST consists of 6670 main cables, of which 150 main cables are connected to the ring beam and fixed on the lug plate. Sensors are installed on these edge main cables and can measure the cable force in them. Therefore, the errors between the surveying value of these main cable forces and the theoretically calculated value can be used to verify whether the model meets the accuracy requirements of the measurement after calibration.

According to China's Cable Structure Technical Specification Code (JGJ 257-2012), after establishing tension in the FAST cable-net structure, the allowable error limit of tension is $\pm 10 \%$. Seeing that the magnetic flux sensor measurement error is approximately $3 \%$ in the 
basic spherical state, if the error between the calculated and measured cable forces of 150 edge main cables is less than 13\%, the MST model has been successfully updated [18].

In October 2019, we first performed a thorough measurement of the data, as mentioned earlier, updated the MST model, and verified the accuracy of the new model. The force of 150 edge main cables was measured in the basic sphere state. Errors of main cable force between the measurement and analysis are indicated in Figure 6. The ambient temperature was about $22{ }^{\circ} \mathrm{C}$ during the measurement.

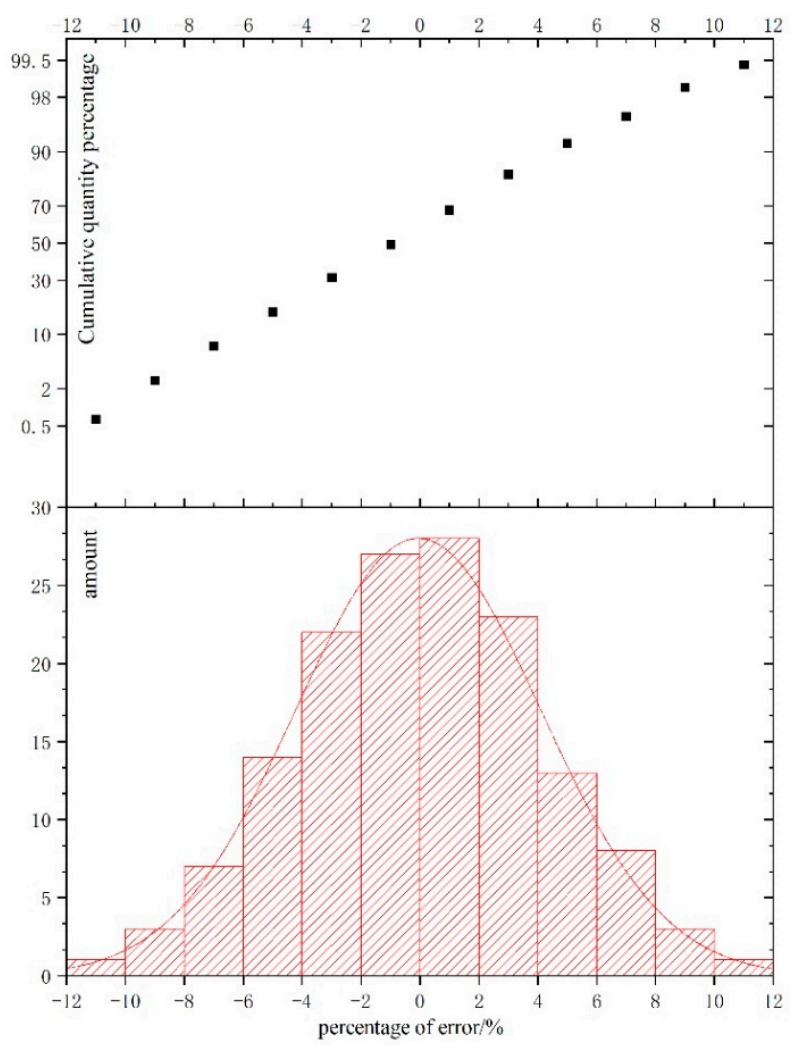

Figure 6. Errors between the measurement and analysis of cable force.

The calculated values of stress in edge main cables are between $629.9 \mathrm{MPa}$ and $732.6 \mathrm{MPa}$, while the values of stress that occurred in analysed cables range from $673.7 \mathrm{MPa}$ to $707.2 \mathrm{MPa}$. As seen from the figure, the maximum error between the measurement and theoretical analysis is $11.3 \%$, while all errors obey normal distribution. This conforms to the standard of the specification. Because the breaking stress of FAST main cables is $1860 \mathrm{MPa}$, the overall cable net is still at a low level of stress and has a high safety factor. This result proves the accuracy of the above-mentioned FAST model update method. The reliability of the new model is verified, which provides a guarantee for the subsequent construction and regular operation of the PHM system.

\section{Pre-Evaluation and Health Monitoring System of FAST}

In order to take advantage of the theoretical results, a PHM system for FAST was developed and derived from the updated MST model. The system consists of a preevaluation system and a health monitoring system. The framework diagram is shown in Figure 7. 


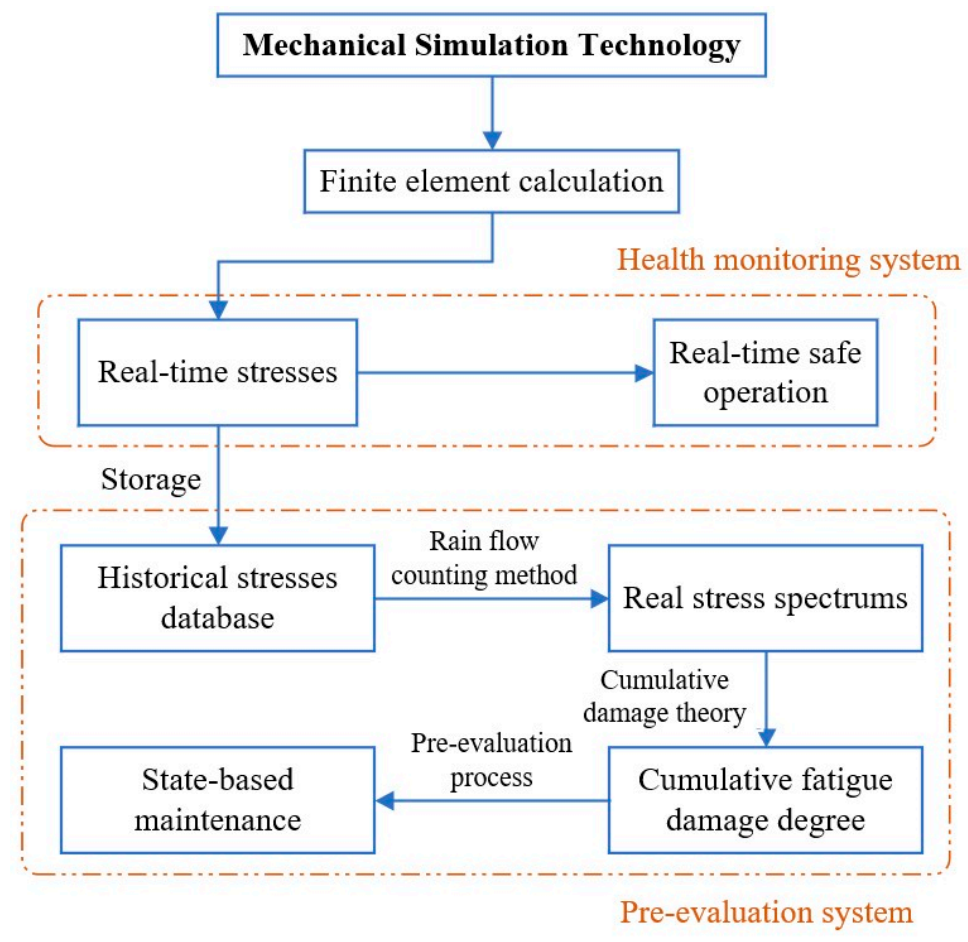

Figure 7. PHM framework of Fast.

\subsection{Pre-Evaluation System}

The rainflow counting method is an efficient method used for fatigue data analysis. It divides the measured historical load data of the structure into several load cycles and estimates the fatigue life of the structure based on these cycles. The fatigue test load spectra are also compiled. This method widely used nowadays in fatigue-life calculations [19].

In the daily observation process of FAST, the obtained stress of each cable is stored in the database in chronological order. Through the rainflow counting method, the fatigueload spectrum analysis of the stress of all cables can be utilized to obtain the actual stress amplitude of all cables. Therefore, the Miner linear cumulative damage theory can be used to obtain the incremental fatigue damage degree to date [20].

Supposing that the fatigue damage of the cable under each stress level is performed independently, the total damage can be linearly superimposed. For a certain level of stress cycles, the number of cycles corresponding to the $i$ th equivalent stress amplitude is recorded as $N_{i}$, and then the damage of one cycle is $1 / N_{i}$, so the total damage of all cycles is:

$$
D=\sum_{i=1}^{n} \frac{1}{N_{i}}
$$

where $D$ is the total fatigue damage suffered by the cable to date. From the perspective of engineering applications, when $D=1$, the corresponding cable-fatigue failure occurs according to the Miner rule. Therefore, according to the force-time curve of each cable, the fatigue life of all cables can be evaluated in advance.

After the analysis, the fatigue stress amplitude of the FAST cable net under working conditions is up to $445 \mathrm{MPa}$. Taking into account the service life of FAST and the complexity of the working conditions, the cables of the structure are required to meet: $1.500 \mathrm{MPa}$ fatigue-stress amplitude; 2 . Upper limit of stress of $0.4 \mathrm{~F}_{\mathrm{ptk}}$, where $\mathrm{F}_{\mathrm{ptk}}$ refers to the standard value of the strength of the cable; 3 . At least two million stress cycles. Once it is discovered that some cables are at risk of fatigue failure, on-site staff can order suitable cables from the manufacturer in advance and replace them at the right moment to ensure the orderly progress of FAST observations. 


\subsection{Health Monitoring System}

In the daily observation process of FAST, through analysis of real-time collected data, the cable force, stress, and other parameters of each component in the structure can be accurately obtained. The safe operating status of the cable network structure can be monitored in real time, and early warning is also provided when a dangerous situation occurs, which makes the real-time health monitoring of FAST possible.

Because each round of the finite element analysis takes approximately 7-8 s (the time will be lower with the update of the hardware), the obtained stress of the cable-net structure reflects the state of the construction $8 \mathrm{~s}$ ago. The piston of the FAST actuator has a maximum operating speed of about $1.6 \mathrm{~mm} / \mathrm{s}$; therefore, in a calculation cycle, the maximum actuator stroke is $\pm 12.8 \mathrm{~mm}$ [21]. According to our calculation, the maximum variation in cable force resulting from this stroke is around $\pm 16 \mathrm{MPa}$, far from the tensile strength (1860 MPa), which means that the stress level of the FAST cable net will not change significantly during this period of time. Therefore, real-time finite element analysis can accurately reflect the internal force distribution of the structure, and it is entirely feasible to establish an effective health monitoring system.

Currently, the arrangement work of the PHM system has been completed on the FAST field to serve this significant project. The system has played a significant role in ensuring the stable observation of FAST. The schematic diagram of the PHM system is described in Figure 8 .

Preevaluation and health monitoring system of FAST cable-net structure
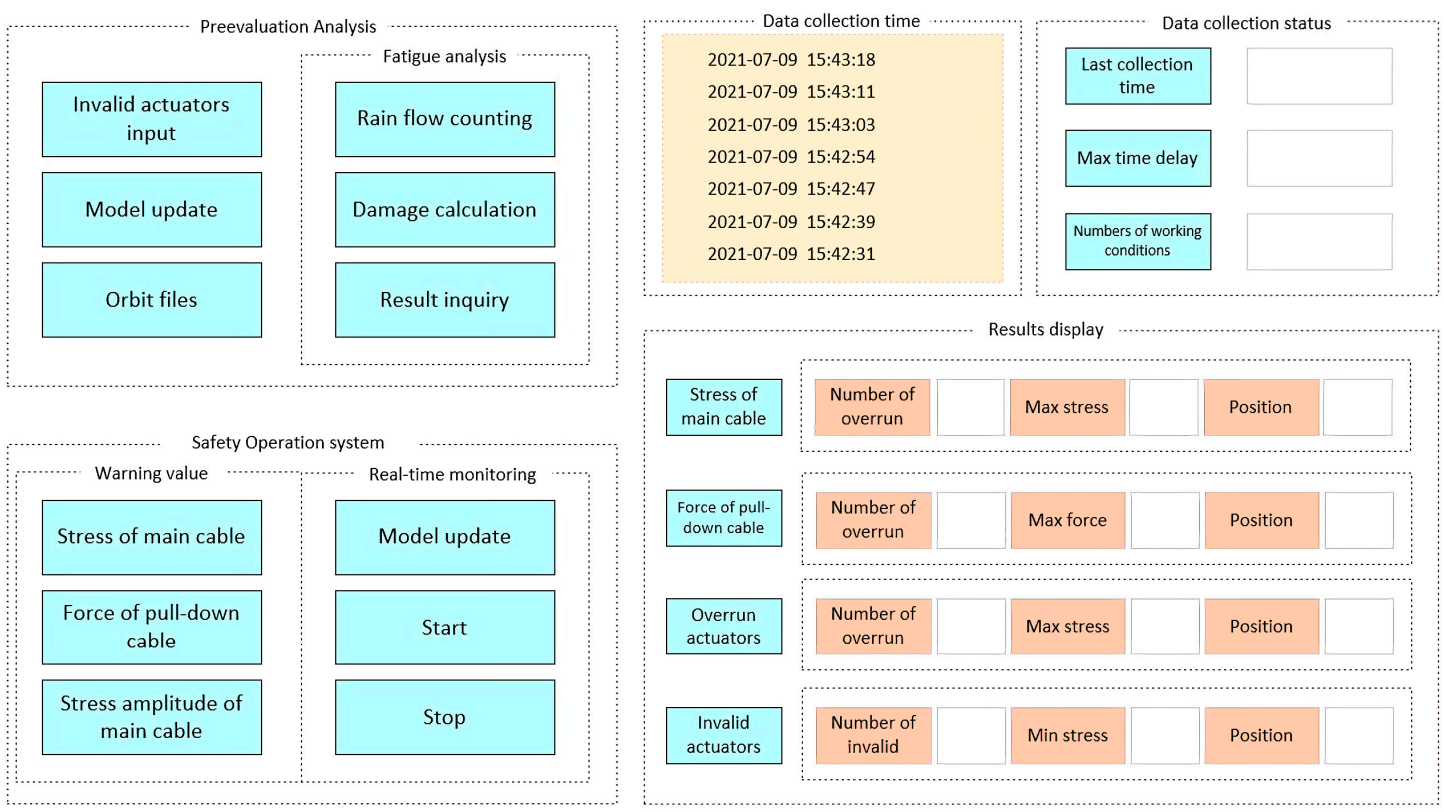

Figure 8. Schematic diagram of PHM system.

\section{Conclusions}

As the largest radio telescope globally, health monitoring of the FAST structure is critical. In order to improve the safety of FAST observations and discover possible safety problems in advance, this paper has obtained the following conclusions through systematic research

1. MST is applicable to the establishment of the FAST model, which can be updated automatically and over time. The model fits in well with the on-site structure.

2. A method to update the FAST model with regularly measured data is proposed, and the field test proves that the updated model is highly reliable. 
3. A PHM system consisting of both pre-evaluation and health monitoring components has been developed to provide a visual and automated early-warning system for the structural health monitoring of FAST, which significantly facilitates the maintenance work of on-site staff. The PHM system has provided vital support for the stable observations of FAST.

\begin{abstract}
Author Contributions: Conceptualization, Y.S. and M.D.; data curation, Y.S.; formal analysis, Y.S.; funding acquisition, B.L. and M.D.; investigation, P.J.; methodology, B.L. and Y.W.; project administration, B.L.; resources, Q.L.; software, Q.L. and P.J.; supervision, B.L. and Y.W.; validation, B.L., Y.W. and M.D.; visualization, Y.S.; writing—original draft, Y.S., B.L., Y.W. and M.D.; writing-review and editing, B.L. and P.J. All authors have read and agreed to the published version of the manuscript.

Funding: This research was funded by the National Natural Science Foundation of China, Grant Numbers 11973006, 51778300 and 52108150; the Key Research and Development Project of Jiangsu Province, Grant Number BE2020703; the Natural Science Foundation of Jiangsu Province, Grant Numbers BK20190753 and BK20191390; the Six Talent Peaks Project of Jiangsu Province, Grant Number JZ-017; and the Natural Science Foundation of the Jiangsu Higher Education.
\end{abstract}

Data Availability Statement: The data presented in this study are available on request from the corresponding author.

Acknowledgments: The authors would like to thank Jianling Li, Lifan Huang, and Hui Li for their constructive contributions to our study and field test.

Conflicts of Interest: The authors declare no conflict of interest.

\title{
References
}

1. Nan, R. Five-hundred-meter aperture spherical radio telescope (FAST). Sci. China Ser. G 2006, 49, 129-148. [CrossRef]

2. Nan, R.; Li, D.I.; Jin, C.; Wang, Q.; Zhu, L.; Zhu, W.; Zhang, H.; Yue, Y.; Qian, L. The Five-hundred-meter Aperture Spherical Radio Telescope (FAST) Project. Int. J. Mod. Phys. D 2012, 20, 989-1024. [CrossRef]

3. Jin, X.; Fan, F.; Qian, H.; Shen, S. Optimal sensor placement of 30-meter scaled structure of large telescope FAST. J. Harbin Inst. Technol. 2009, 41, 31-35.

4. Yao, R.; Zhu, W.; Yang, Q. Dimension optimization design of the Stewart platform in FAST. Adv. Des. Technol. 2011, 308-310, 2110-2113. [CrossRef]

5. Ming, Z.; Wang, Q.; Zheng, L.; Wu, M.; Xue, J. Research for reliability of the actuator of fast reflector based on FMECA. In Proceedings of the 2014 International Conference on Reliability, Maintainability and Safety (ICRMS 2014), Guangzhou, China, 6-8 August 2014.

6. Lu, Y.; Ren, G. Simulation of the Cable Mesh Reflector for the Large Radio Telescope Fast. Eng. Mech. 2007, 24, 165-169.

7. Kong, X.; Jiang, P.; Wang, Q. A Study of Influences of Value Variations of Structural Parameters on Forces in Cables in the Net Structure of Cables of the FAST. Astron. Res. Technol. 2015, 12, 159-165.

8. Li, Q.; Jiang, P.; Li, H. Prognostics and health management of FAST cable-net structure based on digital twin technology. Res. Astron. Astrophys. 2020, 20, 067. [CrossRef]

9. Jiang, P.; Wang, Q.M.; Zhao, Q. Optimization and Analysis on Cable Net Structure Supporting the Reflector of the Large Radio Telescope FAST. Appl. Mech. Mater. 2011, 94-96, 979-982. [CrossRef]

10. Fan, F.; Jin, X.; Qian, H. Fatigue analysis of FAST cable-net structure under long-term active shape-changing work. J. Build. Struct. 2010, 31, 17-23.

11. Luo, B.; Guo, Z.; Wang, K.; Xiao, Q. Performance optimization analysis of FAST reflector supported cable-net based on initial baseline state. China Civ. Eng. J. 2015, 48, 12-22.

12. Sun, X.; Wang, Q.; Zhu, M.; Wu, M. Application of optical fibre Bragg grating strain gauge to cable force monitoring of FAST. Opt. Precis. Eng. 2015, 23, 919-925.

13. Zhu, M.; Yang, L.; Lei, Z.; Wang, Y. Fuzzy Fault Tree Theory-Based Fault Search Strategy Research for FAST Hydraulic Actuators. In Proceedings of the 2016 International Conference on Reliability, Maintainability and Safety (ICRMS 2016), Hangzhou, China, 26-28 October 2016.

14. Zhu, W.; Qin, H.; Li, J.; Ou, J. Monitoring Cable Force of FAST Project Based on Fibre Bragg Grating Sensor External Installed on Anchorage Zone. J. Mech. Eng. 2017, 53, 23-30. [CrossRef]

15. Fan, F.; Wang, H.; Qian, H.; Jin, X.; Chen, M.; Shen, S. Investigation and application of early-warning system for safety of support structure for active reflector of FAST. J. Build. Struct. 2010, 31, 24-31.

16. Shi, Y.; Yao, B.; Zhang, Q.; Mei, X. Study on Numerical Simulation Technology Based on ANSYS of Fracture Behavior in Metal Forming Process. Appl. Mech. Mater. 2011, 130-134, 976. [CrossRef] 
17. Sun, X.; Wang, Q.; Wu, M. Performance Tests of a Monitoring-Management System of Actuators for the FAST. Astron. Res. Technol. 2013, 10, 227-233.

18. Liu, Z.; Jiang, A.; Zhang, A.; Xing, Z.; Du, X. Intelligent Prediction Method for Operation and Maintenance Safety of Prestressed Steel Structure Based on Digital Twin Technology. Adv. Civ. Eng. 2021, 2021, 6640198. [CrossRef]

19. Xu, M.; Yang, C.; Duan, Y.; Wang, Y.; Huang, W.; Ma, F. Fatigue Life Prediction Based on Rain-flow Counting Method. Mach. Des. Res. 2016, 32, 184-187.

20. Wang, H.; Wang, K.; Zhao, L. Random Vibration Fatigue Life Analysis Based on Nonlinear Cumulative Damage. J. Vib. Meas. Diagn. 2018, 38, 991-996.

21. Zhang, H.; Wu, M.; Yue, Y.; Gan, H.; Hu, H.; Huang, S. EMC design for actuators in the FAST reflector. Res. Astron. Astrophys. 2018, 18, 048. [CrossRef] 